\title{
Approximate Shortest Paths in Simple Polyhedra
}

\author{
Fajie $\mathrm{Li}^{1}$ and Reinhard Klette ${ }^{2}$ \\ ${ }^{1}$ College of Computer Science and Technology \\ Huaqiao University, Xiamen, Fujian, China \\ 2 Computer Science Department, The University of Auckland \\ Private Bag 92019, Auckland 1142, New Zealand \\ li.fajie@hqu.edu.cn, r.klette@auckland.ac.nz
}

\begin{abstract}
Since the pioneering work by (Cohen and Kimmel, 1997) on finding a contour as a minimal path between two end points, shortest paths in volume images have raised interest in computer vision and image analysis. This paper considers the calculation of a Euclidean shortest path (ESP) in a three-dimensional (3D) polyhedral space $\Pi$. We propose an approximate $\kappa(\varepsilon) \cdot \mathcal{O}(M|V|)$ 3D ESP algorithm, not counting time for preprocessing. The preprocessing time complexity equals $\mathcal{O}(M|E|+|\mathcal{F}|+|V| \log |V|)$ for solving a special, but 'fairly general' case of the 3D ESP problem, where $\Pi$ does not need to be convex. $V$ and $E$ are the sets of vertices and edges of $\Pi$, respectively, and $\mathcal{F}$ is the set of faces (triangles) of $\Pi . M$ is the maximal number of vertices of a so-called critical polygon, and $\kappa(\varepsilon)=\left(L_{0}-L\right) / \varepsilon$ where $L_{0}$ is the length of an initial path and $L$ is the true (i.e., optimum) path length. The given algorithm solves approximately three (previously known to be) NP-complete or NP-hard 3D ESP problems in time $\kappa(\varepsilon) \cdot \mathcal{O}(k)$, where $k$ is the number of layers in a stack, which is introduced in this paper as being the problem environment. The proposed approximation method has straightforward applications for ESP problems when analyzing polyhedral objects (e.g., in 3D imaging), of for 'flying' over a polyhedral terrain.
\end{abstract}

\section{Introduction and Related Work}

Since the pioneering work in [10] on finding contours as minimal paths between two end points, minimal paths in volume images have raised interest in computer vision and image analysis (for example, [5[11]). In medical image analysis, minimal paths were extracted in 3D images and applied to virtual endoscopy [11]. However, so far, minimal path computation is typically based on the Fast Marching Method which only considers grid points as the possible vertices of the minimal paths; but there exist Euclidean shortest paths such that none of its vertices is a grid point; see, e.g., the example in Section 4 of [17]. Thus, paths detected by the Fast Marching Method cannot be always the exact Euclidean shortest paths.

There already exist several approximation algorithms for 3D ESP calculations, and we briefly recall those. Pioneering the field, [21] presents an

$$
\mathcal{O}\left(n^{4}(m+\log (n / \varepsilon))^{2} / \varepsilon^{2}\right)
$$

algorithm for the general 3D ESP problem, where $n$ is the descriptional complexity of polyhedral scene elements (that is, vertices, edges, and faces of the polyhedron), $\varepsilon$ the 
accuracy of the algorithm, and $m$ the maximum number of bits for representing a single integer coordinate of elements of the polyhedral scene. This was followed by [9], which presents an approximation algorithm for computing an $(1+\varepsilon)$-shortest path from $p$ to $q$ in time

$$
\mathcal{O}\left(n^{2} \lambda(n) \log (n / \varepsilon) / \varepsilon^{4}+n^{2} \log n r \log (n \log r)\right)
$$

where $r$ is the ratio of the Euclidean distance $d_{e}(p, q)$ to the length of the longest edge of any given obstacle, and

$$
\lambda(n)=\alpha(n)^{\mathcal{O}\left(\alpha(n)^{\mathcal{O}(1)}\right)}
$$

where $\alpha(n)=A^{-1}(n, n)$ is the inverse Ackermann function (see, e.g., [18]), which grows very slowly.

Assume a finite set of polyhedral obstacles in $\mathbb{R}^{3}$. Let $p, q$ be two points outside of the union of all obstacles, and $0<\varepsilon<1$. Reference [12] gives an $\mathcal{O}(\log (n / \varepsilon))$ algorithm to compute an $(1+\varepsilon)$-shortest path from $p$ to $q$ such that it does not intersect the interior of any obstacle. However, this algorithm requires a subdivision of $\mathbb{R}^{3}$ which may be computed in $\mathcal{O}\left(n^{4} / \varepsilon^{6}\right)$.

Given a convex partition of the free space, [2] presents an $\mathcal{O}\left(\left(n / \varepsilon^{3}\right)(\log 1 / \varepsilon)(\log n)\right)$ algorithm for the general 3D ESP problem. Recently, [1] proposes algorithms for calculating approximate ESPs amid a set of convex obstacles. For latest results related to surface ESPs, see [4].

Altogether, the task of finding efficient and easy to implement solutions in this field is certainly challenging; see, for example, [19] saying on page 666 the following, when addressing mainly exact solutions: "The problem is difficult even in the most basic Euclidean shortest-path problem ... in a three-dimensional polyhedral domain $\Pi$, and even if the obstacles are convex, or the domain $\Pi$ is simply connected."

Rubberband algorithms provide a general strategy for solving ESP problems. See [16] for a general introduction into rubberband algorithms; they are characterized by sets of steps, defining possible locations of vertices of Euclidean shortest paths, a local optimization strategy, and a termination criterion; for example, see [1622]. This class of algorithms emerged from a particular rubberband algorithm that was proposed in 2000 for calculating shortest paths in 3D image analysis [6]17].

In this paper, we apply a rubberband algorithm to present an approximate

$$
\kappa(\varepsilon) \cdot \mathcal{O}(M|V|)+\mathcal{O}(M|E|+|S|+|V| \log |V|)
$$

algorithm for ESP calculations when $\Pi$ is a (type-2, see Definition 2 below) simply connected polyhedron which is not necessarily convex.

The given algorithm solves approximately three NP-complete or NP-hard 3D ESP problems in time $\kappa(\varepsilon) \cdot \mathcal{O}(k)$, where $k$ is the number of layers in a stack, which is introduced as the problem environment below. Our algorithm has straightforward applications for ESP problems when analyzing polyhedral objects (e.g., in 3D imaging; for the extensive work using geodesics we just cite [26] as one example), or for 'flying' over a polyhedral terrain. The best known result for the latter problem is due to [27] by proposing an $\mathcal{O}((n / \varepsilon)(\log n)(\log \log n))$ algorithm for computing a $\left(2^{(p-1) / p}+\varepsilon\right)$ approximation of an $L_{p}$-shortest path above a polyhedral terrain. 
Section 2 provides necessary definitions and theorems. Section 3 describes our algorithm. Section 4 gives the time complexity of the algorithm. Section 5 illustrates the algorithm by some examples. Section 6 concludes the paper.

\section{Basics}

The algorithm in this paper generalizes a rubberband algorithm which was studied in [17] for computing ESPs in voxel-curves [14], where each voxel is a grid cube.

We denote by $\Pi$ a simple polyhedron (i.e., a compact polyhedral region which is homeomorphic to a unit ball) in the 3D Euclidean space, which is equipped with an $x y z$ Cartesian coordinate system. Let $E$ be the set of edges of $\Pi ; V=\left\{v_{1}, v_{2}, \ldots, v_{n}\right\}$ the set of vertices of $\Pi$. For $p \in \Pi$, let $\pi_{p}$ be the plane which is incident with $p$ and parallel to the $x y$-plane. The intersection $\pi_{p} \cap \Pi$ is a finite set of simple polygons; a singleton (i.e., a set only containing a single point) is considered to be a degenerate polygon.

Definition 1. A simple polygon $P$, being a connected component of $\pi_{p} \cap \Pi$, is called $a$ critical polygon of $\Pi$ (with respect to $p$ ).

Any vertex $p$ defines in general a finite set of critical polygons. The notion of a critical polygon is also generalized as follows: We assume a simply connected (possibly unbounded) polyhedron $\Pi$, and we allow that the resulting (generalized) critical polygons may also be unbounded. For example, a generalized critical polygon may have a vertex at infinity, or it can be the complement of a critical polygon, as specified in Definition 1 (Section 5] will also make use of generalized critical polygons.)

Definition 2. We say that a simple polyhedron $\Pi$ is a type-1 polyhedron iff any vertex $p$ defines exactly one convex critical polygon. We say that a simple polyhedron $\Pi$ is a type-2 polyhedron iff any vertex $p$ defines exactly one simple critical polygon.

Obviously, each type-1 simple polyhedron is also a type- 2 simple polyhedron. Our main algorithm below applies to type-2 simple polyhedra.

In what follows, $\Pi$ is a type- 2 simple polyhedron. For a simple polygon $P$, let $P^{\circ}$ be its topological interior, $P^{\bullet}$ the closure of $P^{\circ}$, and $\partial P=P^{\bullet} \backslash P^{\circ}$ the frontier of $P$. Let $\rho(p, q)$ be a path from $p$ to $q$.

We recall some concepts introduced in [20]. Let $\left(x_{0}, y_{0}, z_{0}\right)$ be a point in 3D space. Let

$$
\begin{aligned}
& S_{1}=\left\{\left(x, y, z_{0}\right): x_{0} \leq x<\infty \wedge y_{0} \leq y<\infty\right\} \\
& S_{2}=\left\{\left(x, y, z_{0}\right):-\infty<x \leq x_{0} \wedge y_{0} \leq y<\infty\right\} \\
& S_{3}=\left\{\left(x, y, z_{0}\right):-\infty<x \leq x_{0} \wedge-\infty<y \leq y_{0}\right\} \\
& S_{4}=\left\{\left(x, y, z_{0}\right): x_{0} \leq x<\infty \wedge-\infty<y \leq y_{0}\right\}
\end{aligned}
$$

$S_{i}$ is called a $q$-rectangle of type $i$, where $i=1,2,3,4$. Furthermore, let $\left(x_{1}, y_{1}, z_{0}\right)$ be a point in 3D space such that $x_{1}>x_{0}$ and $y_{1}>y_{0}$. Let

$$
\begin{aligned}
& S_{h}=\left\{\left(x, y, z_{0}\right):-\infty<x<\infty \wedge y_{0} \leq y \leq y_{1}\right\} \\
& S_{v}=\left\{\left(x, y, z_{0}\right): x_{0} \leq x \leq x_{1} \wedge-\infty<y<\infty\right\}
\end{aligned}
$$




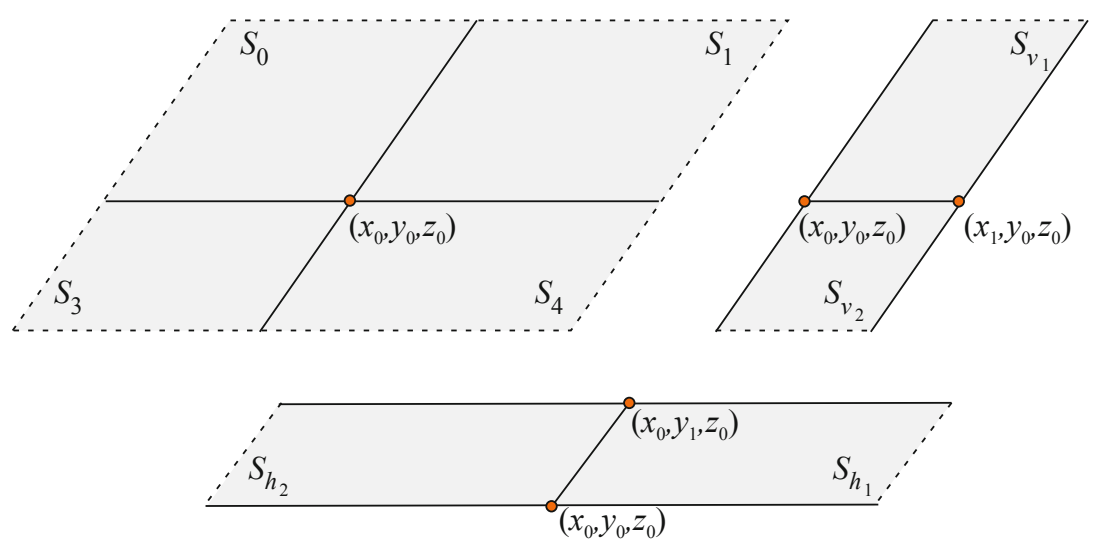

Fig. 1. Axis-aligned rectangles

Finally, let

$$
\begin{aligned}
& S_{h_{1}}=\left\{\left(x, y, z_{0}\right): x_{0} \leq x<\infty \wedge y_{0} \leq y \leq y_{1}\right\} \\
& S_{h_{2}}=\left\{\left(x, y, z_{0}\right):-\infty<x \leq x_{0} \wedge y_{0} \leq y \leq y_{1}\right\} \\
& S_{v_{1}}=\left\{\left(x, y, z_{0}\right): x_{0} \leq x \leq x_{1} \wedge y_{0} \leq y<\infty\right\} \\
& S_{v_{2}}=\left\{\left(x, y, z_{0}\right): x_{0} \leq x \leq x_{1} \wedge-\infty<y \leq y_{0}\right\}
\end{aligned}
$$

$S_{h}, S_{v}, S_{h_{j}}$, and $S_{v_{j}}$ are called horizontal or vertical strips, for $j=1,2$. According to their geometric shape, we notice that

(i) $S_{1}\left[S_{2}, S_{3}, S_{4}\right]$ is unbounded in direction $(+x,+y)[(-x,+y),(-x,-y),(+x,-y)]$;

(ii) $S_{h}\left[S_{v}\right]$ is unbounded in direction $\pm x[ \pm y]$;

(iii) $S_{h_{1}}\left[S_{h_{2}}, S_{v_{1}}, S_{v_{2}}\right]$ is unbounded in direction $+x[-x,+y,-y]$.

$S_{i}, S_{h}, S_{v}, S_{h_{j}}$, and $S_{v_{j}}$ are also called axis-aligned rectangles, where $i=1,2,3,4$ and $j=1,2$. The stack $\mathcal{S}$ of axis-aligned rectangles is called terrain-like if, for at least one of the four directions $-x,+x,-y$, or $+y$, each rectangle in $\mathcal{S}$ is unbounded (see Figure 11.

Let $\left\{s_{1}, s_{2}, \ldots, s_{m}\right\}$ be a set of $m$ line segments and $S$ the union of those segments. Let $p_{1}$ and $p_{2}$ be two different points not in $S$; see Fig. 2. We recall that points in $\mathbb{R}^{3}$ may be sorted by the lexicographic order of their coordinates.

Lemma 1. $d_{e}\left(p_{1}, p\right)+d_{e}\left(p_{2}, p\right)=\min \left\{d_{e}\left(p_{1}, q\right)+d_{e}\left(p_{2}, q\right): q \in S\right\}$ and lexicographic order define a unique point in $S$, which can be computed in $\mathcal{O}(m)$ time.

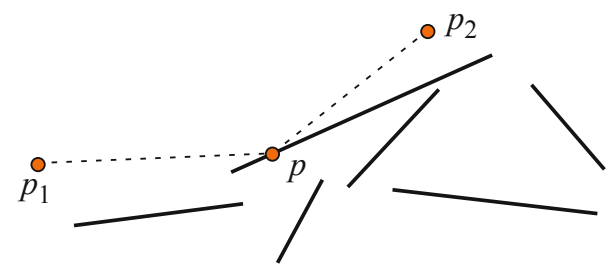

Fig. 2. Two points $p_{1}$ and $p_{2}$ and $m$ segments 
Proof. Consider $m=1$. For line segment $s_{i}$, there is a unique point $q_{i} \in s_{i}$ such that

$$
d_{e}\left(p_{1}, q_{i}\right)+d_{e}\left(p_{2}, q_{i}\right)=\min \left\{d_{e}\left(p_{1}, q\right)+d_{e}\left(p_{2}, q\right): q \in s_{i}\right\}
$$

Consider case $m=2$ and points $q_{1}$ and $q_{2}$. If $d_{e}\left(p_{1}, q_{1}\right)+d_{e}\left(p_{2}, q_{1}\right)<d_{e}\left(p_{1}, q_{2}\right)+$ $d_{e}\left(p_{2}, q_{2}\right)$, then $p=q_{1}$. If $d_{e}\left(p_{1}, q_{1}\right)+d_{e}\left(p_{2}, q_{1}\right)>d_{e}\left(p_{1}, q_{2}\right)+d_{e}\left(p_{2}, q_{2}\right)$, then $p=q_{2}$. If $d_{e}\left(p_{1}, q_{1}\right)+d_{e}\left(p_{2}, q_{1}\right)=d_{e}\left(p_{1}, q_{2}\right)+d_{e}\left(p_{2}, q_{2}\right)$, then we decide for that point which comes first in lexicographic order. Cases $m>2$ follow analogously.

Let $P$ be a convex critical polygon of $\Pi$, defined by the plane $z=c$. Let $p_{1}$ and $p_{2}$ be two points such that their $z$-coordinates satisfy $z_{1}<c<z_{2}$. Then we also have the following (see Theorem 23, page 146, [15]):

Lemma 2. There is a unique point $p \in P^{\bullet}$ such that

$$
d_{e}\left(p_{1}, p\right)+d_{e}\left(p_{2}, p\right)=\min \left\{d_{e}\left(p_{1}, q\right)+d_{e}\left(p_{2}, q\right): q \in P^{\bullet}\right\}
$$

\section{ESP Computation}

We start by presenting a procedure, used by a rubberband algorithm (Algorithm 1 below), and then frequently called in the main algorithm (Algorithm 3 ) of this paper.

Let $\mathcal{F}=\left\{F_{1}, F_{2}, \ldots, F_{m}\right\}$ be the set of all faces of $\Pi$, and $V$ the set of all vertices of $\Pi$. - The following very basic Procedure 1 simply 'walks around' the polyhedron by tracing an intersection with a given plane. We do not detail this procedure; it is a fairly straightforward isoheight trace of a polyhedron, assuming that the data structure of the polyhedron links edges to faces.

Procedure 1. (compute a sequence of vertices of the critical polygon; see Fig. 3) Input: Set $\mathcal{F}$ and a vertex $v \in V$ such that $\pi_{v}$ intersects $\Pi$ in more than just one point. Output: An ordered sequence of all vertices in $V_{v}$, which is the vertex set of the critical polygon $P_{v}$.

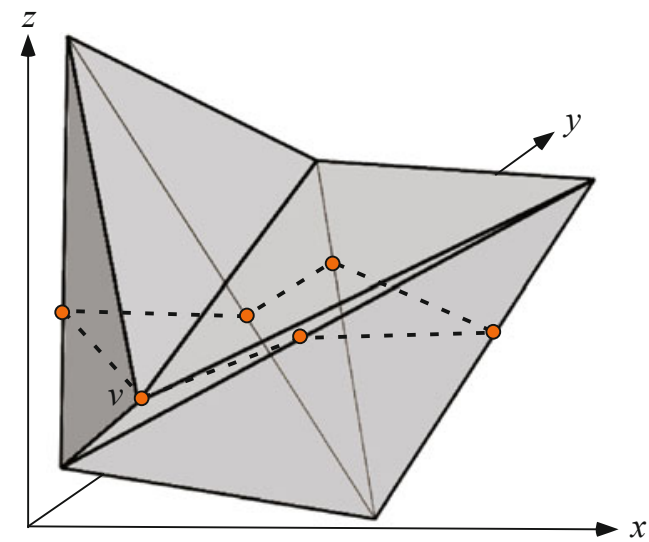

Fig. 3. The labeled vertex $v$ identifies a sequence of six vertices of the critical polygon $P_{v}$, defined by the intersection of plane $\pi_{v}$ with the shown (Schönhardt) polyhedron 
The main ideas of the rubberband algorithm below (Algorithm 1) are as follows: For a start, we randomly take a point in the closure of each critical polygon to identify an initial path from $p$ to $q$. Then we enter a loop; in each iteration, we optimize locally the position of point $p_{1}$ by moving it within its critical polygon, then of $p_{2}, \ldots$, and finally of $p_{k}$. At the end of each iteration, we check the difference between the length of the current path to that of the previous one; if it is less than a given accuracy threshold $\varepsilon>0$ then we stop. Otherwise, we go to the next iteration. - Let $p_{x}$ be the $x$-coordinate of point $p, v_{1_{z}}$ the $z$-coordinate of point $v_{1}$, and so forth.

\section{Algorithm 1. (a rubberband algorithm for type-1 polyhedra)}

Input: Two points $p$ and $q$, a set $\left\{P_{v_{1}}^{\bullet}, P_{v_{2}}^{\bullet}, \ldots, P_{v_{k}}^{\bullet}\right\}$, where $P_{v_{i}}$ is a critical polygon of a given polyhedron $\Pi, k$ vertices $v_{i} \in \partial P_{v_{i}}$ such that $p_{z}<v_{1_{z}}<\cdots<v_{k_{z}}<q_{z}$, for $i=1,2, \ldots, k$, and there is no any other critical polygon of $\Pi$ between $p$ and $q$; given is also an accuracy constant $\varepsilon>0$.

Output: The set of all vertices of an approximate shortest path which starts at $p$, then visits approximate optimal positions $p_{1}, p_{2}, \ldots, p_{k}$ in that order, and finally ends at $q$.

1: For each $i \in\{1,2, \ldots, k\}$, let the initial vertex $p_{i}$ be a vertex of $P_{v_{i}}^{\bullet}$.

2: Let $L_{0}=\infty$. Calculate $L_{1}=\sum_{i=0}^{k} d_{e}\left(p_{i}, p_{i+1}\right)$, where $p_{0}=p$ and $p_{k+1}=q$.

3: while $L_{0}-L_{1} \geq \varepsilon$ do

4: $\quad$ for $i=1,2, \ldots, k$ do

5: $\quad$ Comput $1 \frac{1}{1}$ a point $q_{i} \in P_{v_{i}}^{\bullet}$ such that $d_{e}\left(p_{i-1}, q_{i}\right)+d_{e}\left(q_{i}, p_{i+1}\right)=\min \left\{d_{e}\left(p_{i-1}, p\right)+d_{e}\left(p, p_{i+1}\right): p \in P_{v_{i}}^{\bullet}\right\}$

6: $\quad$ Update the path $\left\langle p, p_{1}, p_{2}, \ldots, p_{k}, q\right\rangle$ by replacing $p_{i}$ by $q_{i}$.

7: $\quad$ end for

8: $\quad$ Let $L_{0}=L_{1}$ and calculate $L_{1}=\sum_{i=0}^{k} d_{e}\left(p_{i}, p_{i+1}\right)$.

9: end while

10: Return $\left\langle p, p_{1}, p_{2}, \ldots, p_{k}, q\right\rangle$.

The set $\left\{P_{v_{1}}^{\bullet}, P_{v_{2}}^{\bullet}, \ldots, P_{v_{k}}^{\bullet}\right\}$ in Algorithm 1 is called the step set of this rubberband algorithm. (Identifying a 'suitable' step set is normally the main issue when defining a rubberband algorithm; see, for example, discussion of step sets in [16 22].)

Theorem 1. If $\Pi$ is a type-1 polyhedron in the input of Algorithm 1 then the solution obtained by Algorithm 1 is an approximate global solution to the 3D ESP problem.

Proof. Let $X=\Pi_{i=1}^{k} P_{u_{i}}^{\bullet} \subset \mathbb{R}^{k}$, where $P_{u_{i}}^{\bullet}$ is as defined in Algorithm 1 As $\Pi$ is a type-1 polyhedron, then $P_{u_{i}}$ is a convex polygon, where $i=1,2, \ldots, k$. Let $Y \subset X$ be the set of all solutions obtained by Algorithm 1, for any initialization in $X$ and the given $\varepsilon>0$.

As each $P_{u_{i}}$ is a convex polygon, by Lemma 2, the point $q_{i}$ in Step 5 of Algorithm 1 is unique, and $q_{i}$ depends continuously upon $p_{i-1}$ and $p_{i+1}$ defined in Step 5 of Algorithm 1. Thus, Algorithm 1 defines a continuous function, denoted by $f$, mapping from $X$ (i.e., an initialization) into $Y$, with values depending on the used accuracy $\varepsilon>0$

\footnotetext{
${ }^{1}$ If $p_{i-1} p_{i+1} \cap P_{v_{i}}^{\circ} \neq \emptyset$, then assign $q_{i} \leftarrow p_{i-1} p_{i+1} \cap P_{v_{i}}^{\circ}$.

${ }^{2}$ If each $P_{u_{i}}^{\bullet}$ is degenerated into a single edge, then there exists a unique solution to the ESP problem; independent of the chosen initialization, solutions will converge to this unique solution if $\varepsilon$ goes to zero; see [8,23,25].
} 
Now let $\bar{v}=\left(v_{1}, v_{2}, \ldots, v_{k}\right) \in Y$. Then $v_{i}$ is either located on an edge of polygon $P_{u_{i}}$, which is contained in the frontier $\partial P_{u_{i}}$, for $i=1,2, \ldots, k$, or $v_{i}$ is located in the interior $P_{u_{i}}^{\circ}$, and $v_{i-1}, v_{i}$ and $v_{i+1}$ are collinear. Thus, $Y$ is a finite set.

It remains to prove that $Y$ is a singleton. Let $\bar{v}_{0} \in Y$; we have that $f^{-1}\left(\bar{v}_{0}\right) \subset X$. For each initialization $\bar{v} \in f^{-1}\left(\bar{v}_{0}\right)$, as $f$ is a continuous function, there exists a sufficiently small open neighborhood (with respect to the usual topology on $\mathbb{R}^{k}$ ) of $\bar{v}$, denoted by $N\left(\bar{v}, \delta_{\bar{v}}\right)$, such that for each $\bar{v}^{\prime} \in N\left(\bar{v}, \delta_{\bar{v}}\right), f\left(\bar{v}^{\prime}\right)=\bar{v}_{0}$. Thus, $N\left(\bar{v}, \delta_{\bar{v}}\right) \subseteq f^{-1}\left(\bar{v}_{0}\right)$ and $\cup_{v \in f^{-1}\left(\bar{v}_{0}\right)} N\left(\bar{v}, \delta_{\bar{v}}\right) \subseteq f^{-1}\left(\bar{v}_{0}\right)$.

On the other hand, because (simply by definition) $f^{-1}\left(\bar{v}_{0}\right)=\left\{\bar{v}: \bar{v}_{0}=f(\bar{v})\right\}$ and $\bar{v} \in N\left(\bar{v}, \delta_{\bar{v}}\right)$, we have that $f^{-1}\left(\bar{v}_{0}\right) \subseteq \cup_{\bar{v} \in f^{-1}\left(\bar{v}_{0}\right)} N\left(\bar{v}, \delta_{\bar{v}}\right)$. Therefore, $f^{-1}\left(\bar{v}_{0}\right)=$ $\cup_{\bar{v} \in f^{-1}\left(\bar{v}_{0}\right)} N\left(\bar{v}, \delta_{\bar{v}}\right)$. Because $N\left(\bar{v}, \delta_{\bar{v}}\right)$ is an open set, $f^{-1}\left(\bar{v}_{0}\right)$ is also open. Let

$$
f^{-1}\left(\bar{v}_{0}\right)=\cup_{i=1}^{k} S_{i}
$$

where $S_{i}$ is an open subset of $P_{u_{i}}^{\bullet}$, for $i=1,2, \ldots, k$. Recall that $f^{-1}\left(\bar{v}_{0}\right) \subset X$, thus there exists an $S_{i}$ such that $\emptyset \subset S_{i} \subset P_{u_{i}}^{\bullet}$.

Without loss of generality, suppose that $\emptyset \subset S_{1} \subset P_{u_{1}}^{\bullet}$. This implies that there exists a point $\left(x_{0}, y_{0}\right) \in P_{u_{1}}^{\bullet}$ such that $\left.\left.\emptyset \subset S_{1}\right|_{x_{0}} \subset P_{u_{1}}^{\bullet}\right|_{x_{0}}$ Thus, $\left.S_{1}\right|_{x_{0}}$ is a nonempty open subset of $\left.P_{u_{1}}^{\bullet}\right|_{x_{0}}$. Set $\left.S_{1}\right|_{x_{0}}$ is a union of a countable number of open or half-open intervals (see Proposition 5.1.4 in [24]).

Thus, there exists a point $\left.w_{1} \in P_{u_{1}}^{\bullet}\right|_{x_{0}} \backslash S_{1}$ such that, for every positive $\varepsilon_{1}$, there exists a point $w_{1}^{\prime} \in N\left(w_{1}, \varepsilon_{1}\right) \cap S_{1}$ [again, $N\left(w_{1}, \varepsilon_{1}\right)$ is an open neighborhood with respect to the usual topology on $P_{u_{1}}^{\bullet}$. Therefore, there exists a point $\bar{v}_{1} \in X \backslash f^{-1}\left(\bar{v}_{0}\right)$ such that, for each positive $\varepsilon_{1}$, there exists a point $\bar{v}_{1}^{\prime} \in N\left(\bar{v}_{1}, \varepsilon_{1}\right) \cap f^{-1}\left(\bar{v}_{0}\right)$. This contradicts that $f$ is a continuous function on $X$. Thus, $Y$ is a singleton.

As an informal interpretation of this proof: If $\varepsilon$ is sufficiently small then $Y$ is a neighborhood $N\left(\bar{v}, \delta_{\bar{v}}\right)$ of a single point $\bar{v}$, where $\delta_{\bar{v}}$ is sufficiently small (depending on $\varepsilon$ ) such that computers regard $N\left(\bar{v}, \delta_{\bar{v}}\right)$ as a single point $\bar{v}$ because of rounding.

If input $\Pi$ is a type- 2 polyhedron then the solution obtained by Algorithm 1 might not be an approximate global solution to the 3D ESP problem. However, following Theorem 1 we propose the following modification of Algorithm 1 for type-2 polyhedrons, with an initial mapping of non-convex polygons on their convex hulls:

Algorithm 2. (a rubberband algorithm for type-2 polyhedra)

Both input and output are the same as in Algorithm 1 .

1: For $i \in\{1,2, \ldots, k\}$, apply (e.g.) the Melkman algorithm for computing $C\left(P_{v_{i}}\right)$, the convex hull of $P_{v_{i}}$.

2: Let $C\left(P_{v_{1}}^{\bullet}\right), C\left(P_{v_{2}}^{\bullet}\right), \ldots, C\left(P_{v_{k}}^{\bullet}\right), p$, and $q$ be the input of Algorithm 1 for computing an approximate shortest route $\left\langle p, p_{1}, \ldots, p_{k}, q\right\rangle$.

3: For $i=1,2, \ldots, k-1$, find a point $q_{i} \in C\left(P_{v_{i}}^{\bullet}\right)$ such that $d_{e}\left(p_{i-1}, q_{i}\right)+d_{e}\left(q_{i}, p_{i+1}\right)=\min \left\{d_{e}\left(p_{i-1}, p\right)+d_{e}\left(p, p_{i+1}\right): p \in C\left(P_{v_{i}}^{\bullet}\right)\right\}$. Update the path for each $i$ by $p_{i}=q_{i}$.

\footnotetext{
$\left.{ }^{3} S\right|_{x_{0}}=\left\{\left(x_{0}, y\right):(x, y) \in S \wedge x=x_{0}\right\}$

${ }^{4}$ If $p_{i-1} p_{i+1} \cap P_{p_{i}}^{\circ} \neq \emptyset$, then set $q_{i} \leftarrow p_{i-1} p_{i+1} \cap P_{p_{i}}^{\circ}$.
} 
4: Let $P_{v_{1}}^{\bullet}, P_{v_{2}}^{\bullet}, \ldots, P_{v_{k}}^{\bullet}, p$ and $q$ be the input of Algorithm 1 and points $p_{i}$ as obtained in Step 3 are the initial vertices $p_{i}$ in Step 1 of Algorithm 1 . Continue with running Algorithm 1 .

5: Return $\left\langle p, p_{1}, \ldots, p_{k-1}, p_{k}, q\right\rangle$ as provided in Step 4.

Step 2 iterates through the closures of convex hulls. The iteration through step sets $C\left(P_{v_{i}}^{\bullet}\right)$ only occurs in Step 4 (i.e., when applying Subalgorithm 1 for a second time, using the same $\varepsilon)$. Algorithm 2 provides an $\left(1+\left(L_{2}-L_{1}\right) / L\right)$-approximate global solution for the ESP, where $L$ is the length of an optimal path; $L_{1}$ is the length of the path obtained in Step 2; $L_{2}$ the length of the final path obtained in Step 5. Note that $L_{2} \geq L_{1}$, and $L_{2}=L_{1}$ if all polygons $P_{i}$ are convex. Also note that $L<L_{1}$, then $\left(1+\left(L_{2}-L_{1}\right) / L\right) \leq L_{2} / L_{1}$. Thus, Algorithm 2 provides an $L_{2} / L_{1}$-approximate global solution for the ESP problem.

The main ideas of Algorithm 3 below are as follows: We apply Procedure 1 to compute the step set of a rubberband algorithm as given in Algorithms 1 or 2. Then we simply apply this rubberband algorithm to compute (of course, approximately only, defined by the chosen accuracy $\varepsilon$ ) the ESP.

For the input polyhedron we assume that it is of type-2. For example, the Schönhardt polyhedron as shown in Fig. 3 is of type-2, but it might be rotated such that the resulting polyhedron is not of type- 2 anymore.

\section{Algorithm 3. (main algorithm)}

Input: Two points $p$ and $q$ in $\Pi$; sets $\mathcal{F}$ and $V$ of faces and vertices of $\Pi$, respectively. Output: The set of all vertices of an approximate shortest path, starting at $p$ and ending at $q$, and contained in $\Pi$.

1: Initialize $V^{\prime} \leftarrow\left\{v: p_{z}<v_{z}<q_{z} \wedge v \in V\right\}$.

2: Sort $V^{\prime}$ according to the $z$-coordinate.

3: We obtain $V^{\prime}=\left\{v_{1}, v_{2}, \ldots, v_{k^{\prime}}\right\} \quad$ with $\quad v_{1 z} \leq v_{2 z} \leq \ldots \leq v_{k^{\prime} z}$.

4: Partition $V^{\prime}$ into pairwise disjoint subsets $V_{1}, V_{2}, \ldots$, and $V_{k}$ such that $V_{i}=\left\{v_{i 1}, v_{i 2}, \ldots, v_{i n_{i}}\right\}$, with $v_{i j_{z}}=v_{i j+1}$, for $j=1,2, \ldots, n_{i}-1$, and $v_{i 1 z}<v_{i+1}{ }_{1 z}$, for $i=1,2, \ldots, k-1$. [That is, this step partitions the set $V^{\prime}$ into some subsets such that the points in the same subset have an identical $z$-coordinate.]

5: Set $u_{i} \leftarrow v_{i 1}$, where $i=1,2, \ldots, k$.

6: Set $V^{\prime \prime} \leftarrow\left\{u_{1}, u_{2}, \ldots, u_{k}\right\}$ (then we have that $u_{1 z}<u_{2 z}<\ldots<u_{k z}$ ).

7: for each $u_{i} \in V^{\prime \prime}$ do

8: Apply Procedure 1 for computing $V_{u_{i}}$ (i.e., a sequence of vertices of the critical polygon $P_{u_{i}}$ ).

9: end for

10: Set $\mathcal{F}_{\text {step }} \leftarrow\left\{P_{u_{1}}^{\bullet}, P_{u_{2}}^{\bullet}, \ldots, P_{u_{k}}^{\bullet}\right.$.

11: Set $P \leftarrow\{p\} \cup V^{\prime \prime} \cup\{q\}$.

12: Apply Algorithm 2 on inputs $\mathcal{F}_{\text {step }}$ and $P$, for computing the shortest path $\rho(p, q)$ inside of $\Pi$.

13: Convert $\rho(p, q)$ into the standard form of a shortest path by deleting all vertices which are not on any edge of $\Pi$ (i.e., delete $p_{i}$ if $p_{i}$ is not on an edge of $P_{u_{i}}$ ).

By the discussions after Algorithm 2 , we have the following 
Theorem 2. Algorithm 3 provides an $L_{2} / L_{1}$-approximate global solution for the ESP problem, where $L_{1}$ is the length of the path obtained in Step 2 of Algorithm 2 ; $L_{2}$ is the length of the final path obtained in Step 5 of Algorithm 2

\section{Time Complexity}

At first, we can show (calculation of upper time bounds for involved steps is fairly straightforward) that Procedure 1 can be computed in $\mathcal{O}\left(\left|V_{v}\right|\left|E\left(S_{v}\right)\right|+|\mathcal{F}|\right)$ time, where $S_{v}=\left\{F: F \in \mathcal{F} \wedge e=u w \in E(F) \wedge\left(u_{z} \leq v_{z} \leq w_{z} \vee w_{z} \leq v_{z} \leq u_{z}\right)\right\}$. Then we can show that the time complexity of Algorithm 1 equals $\kappa(\varepsilon) \cdot \mathcal{O}\left(\sum_{j=1}^{k}\left|V_{v_{j}}\right|\right)$, where $\kappa(\varepsilon)$ is the number of iterations of the while loop in Algorithm 1 By Lemma 1 Step 5 can be computed in $\mathcal{O}\left(\left|V_{v_{j}}\right|\right)$ time, where $V_{v_{j}}$ is as in Algorithm 1, for $j=1$, $2, \ldots, k$. Thus, each iteration of Algorithm 1 can be computed in $\mathcal{O}\left(\sum_{j=1}^{k}\left|V_{v_{j}}\right|\right)$ time. Obviously, Algorithm 2 has the same time complexity as Algorithm 1 - These three results allow us then to show that Algorithm 3 can be computed in

$$
\kappa(\varepsilon) \cdot \mathcal{O}\left(\sum_{j=1}^{k}\left|V_{u_{j}}\right|\right)+\mathcal{O}\left(\sum_{j=1}^{k}\left|V_{u_{j}}\right|\left|E\left(S_{u_{j}}\right)\right|+|\mathcal{F}|+|V| \log |V|\right)
$$

where the second term is the time for preprocessing. This can finally be reformulated in the more compact form that Algorithm 3 is of complexity

$$
\kappa(\varepsilon) \cdot \mathcal{O}(M|V|)+\mathcal{O}(M|E|+|\mathcal{F}|+|V| \log |V|)
$$

for $M=\max \left\{\left|V_{u_{j}}\right|: j=1,2, \ldots, k\right\}$, where the second $\mathcal{O}(\ldots)$ term is the time for preprocessing.

In Algorithm 1, let $\kappa(\varepsilon)=\frac{L_{0}-L}{\varepsilon}$ be a function which only depends upon the difference between the lengths $L_{0}$ of an initial path and $L$ of the optimum path, and the accuracy constant $\varepsilon$. Let $L_{m}$ be the length of the $m$-th updated path, for $m=0,1,2, \ldots$, with $L_{m}-L_{m+1} \geq \varepsilon$ (otherwise the algorithm stops). It follows that

$$
\kappa(\varepsilon)=\frac{L_{0}-L}{\varepsilon} \geq 1+\frac{L_{1}-L}{\varepsilon} \geq \cdots \geq m+\frac{L_{m}-L}{\varepsilon}
$$

The sequence $\left\{m+\frac{L_{m}-L}{\varepsilon}\right\}$ is monotonously decreasing, lower bounded by 0 , and stops at the first $m_{0}$ where $L_{m_{0}}-L_{m_{0}+1}<\varepsilon$.

We have implemented a simplified version of Algorithm 1 where all $P_{v_{i}}^{\bullet}$ s were degenerated to be line segments. Thousands of experimental results indicated that $\kappa(\varepsilon)$ does not depend on the number $k$ of segments but the value of $\varepsilon$. We selected $\varepsilon=10^{-15}$ and $k$ was in between 4 and 20,000, the observed maximal value of $\kappa(\varepsilon)$ was 380,000. It shows that the smallest upper bound of $\kappa(\varepsilon) \geq \kappa\left(10^{-15}\right) \geq 380,000$. In other words, the number of iterations in the while-loop can be huge even for some small value of $k$. On the other hand, all these experimental results indicated that $\left|L_{m}-L_{m+1}\right| \leq 1.2$, when $m>200$ and $L$ was between 10,000 and 2,000,000. It showed that $\kappa(1.2) \leq 200$ and the relative error $\left|L_{m}-L_{m+1}\right| / L \leq 1.2 \times 10^{-4}$. In other words, these experiments showed that the algorithm already reached an approximate ESP with a very minor relative error after 200 iterations of the while loop; the remaining iterations were 'just' spent on improving a very small fraction of the length of the path. 


\section{Examples: Three NP-Complete or NP-Hard Problems}

We apply Algorithms 2 and 3 for approximate solutions of hard problems, characterized below (by appropriate references) as being NP-complete or NP-hard. Let $p, q \in \Pi$ such that $p_{z}<q_{z}$. Let $V_{p q}=\left\{v: p_{z}<v_{z}<q_{z} \wedge v \in V\right\}$, where $V$ is the set of all vertices of $\Pi$. For doing so, we are allowing for input polyhedra different from the bounded type2 polyhedra so far, but only input polyhedra which allow us to use those algorithms without any further modification.

We consider unbounded polyhedra (which also satisfy the type- 2 constraint), and, thus, generalized critical polygons.

Example 1. Let $\Pi$ be a simply connected polyhedron such that each critical polygon is the complement of an axis-aligned rectangle. Following Section 4 the Euclidean shortest path between $p$ and $q$ inside of $\Pi$ can be approximately computed in $\kappa(\varepsilon) \cdot \mathcal{O}\left(\left|V_{p q}\right|\right)$ time. Therefore, the 3D ESP problem can be approximately solved efficiently in such a special case. Finding the exact solution is NP-complete because of the following

Theorem 3. ([20], Theorem 4) It is NP-complete to decide wether there exists an obstacle-avoiding path of Euclidean length at most L among a set of stacked axisaligned rectangles (see Fig. 4). The problem is (already) NP-complete for the special case that the axis-aligned rectangles are all q-rectangles of types 1 or 3.

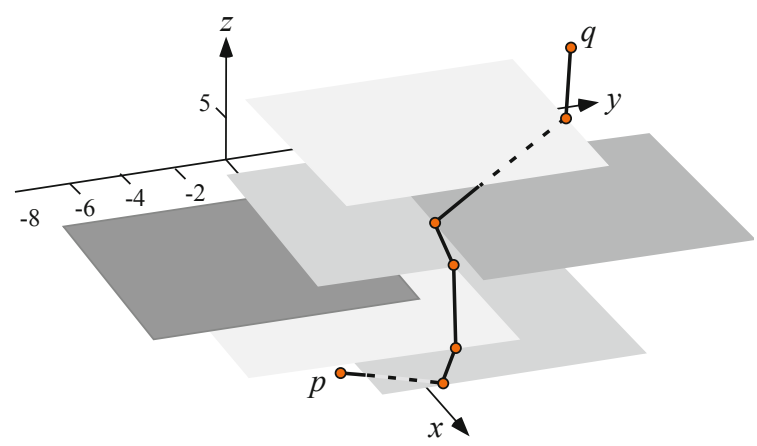

Fig. 4. A path from $p$ to $q$ which does not intersect any of the shown rectangles at an inner point

Example 2. Let $\Pi$ be a simply connected polyhedron such that each critical polygon is the complement of a triangle. Following Section 4 the Euclidean shortest path between $p$ and $q$ inside of $\Pi$ can be approximately computed in $\kappa(\varepsilon) \cdot \mathcal{O}\left(\left|V_{p q}\right|\right)$ time. Finding the exact solution is NP-hard because of the following

Theorem 4. ([7]) It is NP-hard to decide whether there exists an obstacle-avoiding path of Euclidean length at most L among a set of stacked triangles.

Example 3. Let $\mathcal{S}$ be a stack of $k$ horizontal or vertical strips. The Euclidean shortest path among $\mathcal{S}$ can be approximately computed in $\kappa(\varepsilon) \cdot \mathcal{O}(k)$ time. Finding the exact solution is NP-complete because of the following 
Theorem 5. ([20], Theorem 5) It is NP-complete to decide whether there exists an obstacle-avoiding path of Euclidean length at most L among a finite number of stacked horizontal and vertical strips.

Example 4. Let $\mathcal{S}$ be a stack of $k$ terrain-like axis-parallel rectangles. The Euclidean shortest path among $\mathcal{S}$ can be approximately computed in $\kappa(\varepsilon) \cdot \mathcal{O}(k)$ time. The best known algorithm for finding the exact solution has a time complexity in $\mathcal{O}\left(k^{4}\right)$ due to the following

Theorem 6. ([20], Theorem 6) Let $\mathcal{S}$ be a stack of $k$ terrain-like axis-parallel rectangles. The Euclidean shortest path among $\mathcal{S}$ can be computed in $\mathcal{O}\left(k^{4}\right)$ time.

\section{Conclusions}

This paper described an algorithm for solving the 3D ESP problem when the domain $\Pi$ is a type-2 simply connected polyhedron; the algorithm has a time complexity in $\kappa(\varepsilon) \cdot \mathcal{O}(M|V|)+\mathcal{O}(M|E|+|\mathcal{F}|+|V| \log |V|)($ where $\mathcal{O}(M|E|+|\mathcal{F}|+|V| \log |V|)$ is the time for preprocessing). It was also shown that the algorithm approximately solves three NP-complete or NP-hard problems in time $\kappa(\varepsilon) \cdot \mathcal{O}(k)$, where $k$ is the number of layers in the given stack of polygons.

Our algorithm has straightforward applications on ESP problems in 3D imaging (where proposed solutions depend on geodesics), or when 'flying' over a polyhedral terrain. The best result so far for the latter problem was an $\mathcal{O}((n / \varepsilon)(\log n)(\log \log n))$ algorithm for computing a $\left(2^{(p-1) / p}+\varepsilon\right)$-approximation to the $L_{p}$-shortest path above a polyhedral terrain.

As there does not exist an algorithm for finding exact solutions to the general 3D ESP problem (see Theorem 9, [3]), our method defines a new opportunity to find approximate (and efficient!) solutions to the discussed classical, fundamental, hard and general problems.

\section{References}

1. Agarwal, P.K., Sharathkumar, R., Yu, H.: Approximate Euclidean shortest paths amid convex obstacles. In: Proc. ACM-SIAM Sympos. Discrete Algorithms, pp. 283-292 (2009)

2. Aleksandrov, L., Maheshwari, A., Sack, J.-R.: Approximation algorithms for geometric shortest path problems. In: Proc. ACM Sympos. Theory Comput., pp. 286-295 (2000)

3. Bajaj, C.: The algebraic complexity of shortest paths in polyhedral spaces. In: Proc. Allerton Conf. Commun. Control Comput., pp. 510-517 (1985)

4. Balasubramanian, M., Polimeni, J.R., Schwartz, E.L.: Exact geodesics and shortest paths on polyhedral surfaces. IEEE Trans. Pattern Analysis Machine Intelligence 31, 1006-1016 (2009)

5. Benmansour, F., Cohen, L.D.: Fast object segmentation by growing minimal paths from a single point on 2D or 3D images. J. Math. Imaging Vision 33, 209-221 (2009)

6. Buelow, T., Klette, R.: Rubber band algorithm for estimating the length of digitized spacecurves. In: Proc. ICPR, vol. III, pp. 551-555. IEEE, Los Alamitos (2000)

7. Canny, J., Reif, J.H.: New lower bound techniques for robot motion planning problems. In: Proc. IEEE Conf. Foundations Computer Science, pp. 49-60 (1987) 
8. Choi, J., Sellen, J., Yap, C.-K.: Precision-sensitive Euclidean shortest path in 3-space. In: Proc. ACM Sympos. Computational Geometry, pp. 350-359 (1995)

9. Clarkson, K.L.: Approximation algorithms for shortest path motion planning. In: Proc. ACM Sympos. Theory Comput., pp. 56-65 (1987)

10. Cohen, L.D., Kimmel, R.: Global minimum for active contour models: a minimal path approach. Int. J. Computer Vision 24, 57-78 (1997)

11. Deschamps, T., Cohen, L.D.: Fast extraction of minimal paths in 3D images and applications to virtual endoscopy. Med. Image Anal. 5, 281-299 (2001)

12. Har-Peled, S.: Constructing approximate shortest path maps in three dimensions. In: Proc. ACM Sympos. Computational Geometry, pp. 125-130 (1998)

13. Klette, R., Rosenfeld, A.: Digital Geometry. Morgan Kaufmann, San Francisco (2004)

14. Li, F., Klette, R.: The Class of Simple Cube-Curves Whose MLPs Cannot Have Vertices at Grid Points. In: Andrès, É., Damiand, G., Lienhardt, P. (eds.) DGCI 2005. LNCS, vol. 3429, pp. 183-194. Springer, Heidelberg (2005)

15. Li, F., Klette, R.: Exact and approximate algorithms for the calculation of shortest paths. IMA Minneapolis, Report 2141(2006), www . ima . umn . edu/preprints / oct2006

16. Li, F., Klette, R.: Rubberband algorithms for solving various $2 \mathrm{D}$ or 3D shortest path problems. In: Proc. Computing: Theory and Applications, The Indian Statistical Institute, Kolkata, pp. 9-18. IEEE, Los Alamitos (2007)

17. Li, F., Klette, R.: Analysis of the rubberband algorithm. Image Vision Computing 25, 1588 1598 (2007)

18. Liu, Y.A., Stoller, S.D.: Optimizing Ackermann's function by incrementalization. In: Proc. ACM SIGPLAN Sympos. Partial Evaluation Semantics-Based Program Manipulation, pp. 85-91 (2003)

19. Mitchell, J.S.B.: Geometric shortest paths and network optimization. In: Sack, J.-R., Urrutia, J. (eds.) Handbook of Computational Geometry, pp. 633-701. Elsevier, Amsterdam (2000)

20. Mitchell, J.S.B., Sharir, M.: New results on shortest paths in three dimensions. In: Proc. ACM Sympos. Computational Geometry, pp. 124-133 (2004)

21. Papadimitriou, C.H.: An algorithm for shortest path motion in three dimensions. Inform. Processing Letters 20, 259-263 (1985)

22. Pan, X., Li, F., Klette, R.: Approximate shortest path algorithms for sequences of pairwise disjoint simple polygons. In: Proc. Canadian Conf. Computational Geometry, Winnipeg, Canada, pp. 1-4 (2010)

23. Sharir, M., Schorr, A.: On shortest paths in polyhedral spaces. SIAM J. Comput. 15, 193-215 (1986)

24. Wachsmuth, B.G.: Interactive real analysis, http://web01.shu.edu/projects/ reals/topo/index.html (last visit: May, 2009)

25. Yap, C.-K.: Towards exact geometric computation. Computational Geometry: Theory Applications 7, 3-23 (1997)

26. Wang, Y., Peterson, B.S., Staib, L.H.: 3D brain surface matching based on geodesics and local geometry. Computer Vision Image Understanding 89, 252-271 (2003)

27. Zadeh, H.Z.: Flying over a polyhedral terrain. Inform. Processing Letters 105, 103-107 (2008) 